\title{
Wiener Polarity Index Calculation of Square-Free Graphs and Its Implementation to Certain Complex Materials
}

\author{
Lin Zhang, ${ }^{1}$ Tian-Le Sun $\left(\mathbb{D},{ }^{2}\right.$ Micheal Arockiaraj $\mathbb{D},{ }^{3}$ M. Arulperumjothi $\left(\mathbb{D},{ }^{4}\right.$ \\ and S. Prabhu $\mathbb{D}^{5}$ \\ ${ }^{1}$ School of Library, Anhui Jianzhu University, Hefei 230601, China \\ ${ }^{2}$ College of Economics, Sichuan Agricultural University, Chengdu 610000, China \\ ${ }^{3}$ Department of Mathematics, Loyola College, Chennai 600034, India \\ ${ }^{4}$ Department of Mathematics, Loyola College, University of Madras, Chennai 600034, India \\ ${ }^{5}$ Department of Mathematics, Sri Venkateswara College of Engineering, Sriperumbudur, Kanchipuram 602117, India
}

Correspondence should be addressed to S. Prabhu; drsavariprabhu@gmail.com

Received 23 October 2020; Revised 10 November 2020; Accepted 24 November 2020; Published 23 February 2021

Academic Editor: Hijaz Ahmad

Copyright ( $\odot 2021$ Lin Zhang et al. This is an open access article distributed under the Creative Commons Attribution License, which permits unrestricted use, distribution, and reproduction in any medium, provided the original work is properly cited.

\begin{abstract}
Molecular topology is a portion of mathematical chemistry managing the logarithmic portrayal of chemical materials, permitting a tremendous yet straightforward characterization of the compounds. Concerning the traditional physical-chemical descriptors, it is conceivable to set up direct quantitative structure-activity relationship methods to associate with such descriptors termed topological indices. In this study, we have developed the mathematical technique to study the Wiener polarity index of chemical materials without squares. We have taken the cancer treatment drugs such as lenvatinib and cabozantinib to illustrate our approach. In addition, we explored the inherent property of silicate, Sierpiński, and octahedral-related complex materials that the edge set can be decomposed in such a way that any edge in the same part of the decomposition has an equal number of neighboring vertices and applied the technique to derive the formulae for these materials.
\end{abstract}

\section{Introduction}

Drug discovery is the procedure through which potential new helpful substances are established by utilizing a combination of computational, experimental, translational, and clinical models. Regardless of advances in biotechnology and comprehension of organic frameworks, drug discovery is as yet an extensive, costly, complicated, and inefficient process with a high attrition rate of new therapeutic discovery. Drug design is the inventive process of finding new drugs depending on the information of a biological target. Currently, there are several incredible methodologies for drug design and drug database screening [1-3]. The quantitative structure activity and property relationships (QSAR/QSPR) are mathematical models that endeavor to transmit the structure-derived features of a compound to its biological or physico-chemical movement. The relevance of QSAR/QSPR advance in scientific research starts with the idea of the assortment of the input data from databases, then the explanation of molecular descriptors which describes important information of the composed molecules, and through an explicit method to decrease the number of descriptors to the most instructive descriptors, and the last part is the chemoinformatic tools which are used to assemble models that describe the pragmatic relationship between the structure and property or activity.

A significant development in the automated computer treatment of chemical materials and QSAR has been the application of a numerical procedure, namely, graph theory to chemistry. In chemical graph theory, molecular structures are represented as hydrogen-suppressed graphs, regularly known as molecular graphs, in which the atoms are taken by vertices and the bonds by edges. The associations between molecules can be portrayed by different kinds of topological matrices, for example, distance or adjacency matrices, which can be scientifically converted into real numbers and called 
topological indices. Topological indices (TIs) are usually considered the atomic arrangement of compounds such as atomic size, shape, branching, nearness of heteroatoms, and various bonds [6-10]. The handiness of TIs in QSPR and QSAR contemplates has been broadly illustrated [4], and they likewise have been utilized as a proportion of auxiliary structural similarity or diversity by their application to databases created by PC. The idea of topological indices originated from the work of Wiener, while he was functioning on the paraffin boiling points using Wiener and Wiener polarity indices [5].

All the graphs argued in this paper are simple and finite. Let $G=(V(G), E(G))$ be a connected graph. The cardinality of the vertex set and the edge set of $G$ is represented by $|V(G)|$ and $|E(G)|$, respectively. The number of pentagons and hexagons of $G$ is denoted by $N_{p}(G)$ and $N_{h}(G)$, respectively. For any positive integer $i$, we represent $N_{G}^{i}(v)=$ $\left\{u \in V(G): d_{G}(u, v)=i\right\}$ as the $i^{\text {th }}$ neighborhood of $v$, and thus, clearly the open neighborhood of $v$ (denoted by $N_{G}(v)$ ) is $N_{G}^{1}(v)$, and the degree of $v$ (denoted by $d_{v}$ ) is $\left|N_{G}^{1}(v)\right|$. The first and second Zagreb indices of a graph $G$ are defined, respectively, as follows:

$$
\begin{aligned}
& M_{1}(G)=\sum_{u v \in E(G)}\left[d_{u}+d_{v}\right]=\sum_{v \in V(G)} d_{v}^{2}, \\
& M_{2}(G)=\sum_{u v \in E(G)}\left[d_{u} \times d_{v}\right] .
\end{aligned}
$$

The Wiener polarity index of a graph $G$ is defined as

$$
W_{P}(G)=\left|\left\{\{u, v\}: d_{G}(u, v)=3, u, v \in V(G)\right\}\right| \text {. }
$$

It was observed that $W_{P}(G)=(1 / 2) \sum_{v \in V(G)}\left|N_{G}^{3}(v)\right|[11]$, and the importance of $W_{P}$ has been demonstrated in various papers $[9,10,12-28]$. In this paper, we study the Wiener polarity index of $C_{4}$-free graphs and implement our technique to cancer treatment drugs, silicate, Sierpiński, and octahedral-related networks.

\section{Derivation of the Key Result}

For a tree $T$, it was realized [18] that $W_{P}(T)=\sum_{u v \in E(T)}\left(d_{u}-1\right) \times\left(d_{v}-1\right)$, and interms of Zagreb indices can be rewritten as $W_{P}(T)=M_{2}(T)-M_{1}(T)+|E(T)|$. For a graph $G$, where $G$ is $C_{3}$-free and $C_{4}$-free such that its different cycles have at most one common edge [14], $W_{P}(G)=M_{2}(G)-$ $M_{1}(G)+|E(G)|-5 N_{P}(G)-3 N_{h}(G)$. Suppose $G$ is the $C_{3}$-free graph such that its different cycles have at most one common edge [29], $\quad W_{P}(G)=\quad M_{2}(G)-$ $M_{1}(G)+|E(G)|-5 N_{p}(G)-3 N_{h}(G)-\sum_{i}\left(K_{i}-4\right) N_{q_{i}}(G)$, where $N_{q_{i}}(G)$ is the number of quadrangles of type $i$ such that the sum of degrees on the vertices of that type quadrangle is $K_{i}$. In this section, we fill a gap by computing Wiener polarity indices of $C_{4}$-free graphs.

Theorem 1. Let $G$ be a $C_{4}$-free graph such that its different cycles have at most one common edge. For $u v \in E(G)$, let $k_{u v}=\left|N_{G}(u) \cap N_{G}(v)\right|$. Then,

$$
\begin{aligned}
W_{P}(G)= & \sum_{u v \in E(G)}\left(d_{u}-k_{u v}-1\right) \times\left(d_{v}-k_{u v}-1\right) \\
& -5 N_{p}(G)-3 N_{h}(G) .
\end{aligned}
$$

Proof. Suppose that, for any edge $u v$ of $G$ that $k_{u v}=0$, it is easy to see that $[14,29] \quad W_{P}(G)=\sum_{u v \in E(G)}\left(d_{u}-1\right) \times$ $\left(d_{v}-1\right)-5 N_{P}(G)-3 N_{h}(G)$. Assume that there exists an edge $u v \in E(G)$ such that $k_{u v} \neq 0$, as shown in Figure 1 . Then, the number of pairs of vertices $\{x, y\}$ of $G$ such that $d_{G}(x, y)=3$ and containing the edge $u v$ as the internal edge is $\left|\left\{\left(x_{i}, y_{j}\right): 1 \leq i \leq p, 1 \leq j \leq q\right\}\right|$. Since $d_{u}=p+k_{u v}+1$ and $d_{v}=q+k_{u v}+1$, we have $p q=\left(d_{u}-k_{u v}-1\right)\left(d_{v}-k_{u v}-1\right)$. Hence,

$$
W_{P}(G)=\sum_{u v \in E(G)}\left(d_{u}-k_{u v}-1\right)\left(d_{v}-k_{u v}-1\right)-5 N_{p}(G)-3 N_{h}(G) .
$$

Corollary 1. Let $G$ be a $C_{4}$-free graph such that its different cycles have at most one common edge. If every edge uv $\in E(G)$ such that $k_{u v}=k \geq 0$, then

$$
\begin{aligned}
W_{P}(G)= & M_{2}(G)-(k+1) M_{1}(G)+(k+1)^{2}|E(G)| \\
& -5 N_{p}(G)-3 N_{h}(G) .
\end{aligned}
$$

\section{Numerical Computation}

In this section, we compute the numerical quantities for Wiener polarity indices of lenvatinib and cabozantinib cancer drugs and chemical materials based on the silicate, Sierpiński, and octahedral frameworks.

3.1. Cancer Treatment Drugs. Lenvatinib and cabozantinib [30] are orally accessible multikinase inhibitor and antineoplastic specialist that are utilized in the treatment of advanced, metastatic medullary thyroid cancer (MTC) and hepatocellular carcinoma (HCC). Lenvatinib is a member of the class of quinolines, that is, the carboxamide of 4-3chloro-4-[(cyclopropylcarbamoyl)amino]phenoxy-7-

methoxyquinoline-6-carboxylic acid. Cabozantinib is a dicarboxylic acid diamide, that is, N-phenyl-N'-(4-fluorophenyl)cyclopropane-1,1-dicarboxamide in which the hydrogen at position 4 on the phenyl ring is substituted by an (6,7-dimethoxyquinolin-4-yl)oxy group. The molecular graph structures of lenvatinib and cabozantinib are shown in Figure 2.

Theorem 2. Let $G_{1}$ and $G_{2}$ be the graphs of lenvatinib and cabozantinib. Then, $W_{P}\left(G_{1}\right)=48$, and $W_{P}\left(G_{2}\right)=62$.

Proof. Since lenvatinib and cabozantinib are square-free graphs, we complete the proof by using Theorem 1 and Table 1. Let $E_{i j}=\left\{u v \in E\left(G_{1}\right) ; d_{u}=i, d_{v}=j\right\}$ such that $E\left(G_{1}\right)=\cup_{1 \leq i<j \leq 3} E_{i j}$ : 


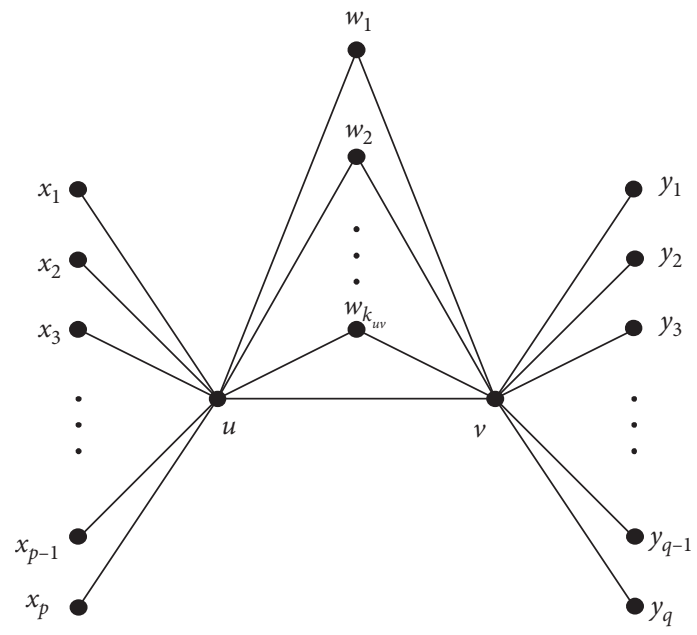

Figure 1: Edge $u v$ of $G$ such that $k_{u v} \neq 0$.<smiles>COc1cc2nccc(Oc3ccc(NC(=O)NC4CC4)c(Cl)c3)c2cc1C(N)=O</smiles>

(a)<smiles>COc1cc2nccc(Oc3ccc(NC(=O)C4(C(=O)Nc5ccc(F)cc5)CC4)cc3)c2cc1OC</smiles>

(b)

FIgure 2: (a) Lenvatinib $\mathrm{C}_{21} \mathrm{H}_{19} \mathrm{ClN}_{4} \mathrm{O}_{4}$. (b) Cabozantinib $\mathrm{C}_{28} \mathrm{H}_{24} \mathrm{FN}_{3} \mathrm{O}_{5}$.

TABLE 1: Edge partition and number of hexagons.

\begin{tabular}{lccccccc}
\hline Graph $G$ & \multicolumn{5}{c}{ Edge partition of $G_{i}$} \\
& $(1,3)$ & $(2,2)$ & $(2,3)$ & $(3,3)$ & $(1,2)$ & $(3,4)$ & $(2,4)$ \\
\hline$G_{1} \cong \mathrm{C}_{21} \mathrm{H}_{19} \mathrm{ClN}_{4} \mathrm{O}_{4}$ & 4 & 4 & 19 & 5 & 1 & - & - \\
$G_{2} \cong \mathrm{C}_{28} \mathrm{H}_{24} \mathrm{FN}_{3} \mathrm{O}_{5}$ & 3 & 7 & 22 & 3 & 2 & 2 & 2 \\
\hline
\end{tabular}

$$
\begin{aligned}
W_{P}\left(G_{1}\right) & =\sum_{u v \in E_{i j}}\left(d_{u}-k_{u v}-1\right)\left(d_{v}-k_{u v}-1\right)-5 N_{p}\left(G_{1}\right)-3 N_{h}\left(G_{1}\right), \\
& =\sum_{\substack{u v \in E_{i j} \\
1 \leq i<j \leq 3 \\
k_{u v}=0}}\left(d_{u}-k_{u v}-1\right)\left(d_{v}-k_{u v}-1\right)+\sum_{\substack{u v \in E_{i j} \\
1 \leq i<j \leq 3 \\
k_{u v}=1}}\left(d_{u}-k_{u v}-1\right)\left(d_{v}-k_{u v}-1\right)-3(3), \\
& =48 .
\end{aligned}
$$


In a similar way, we can complete the proof of cabozantinib.

3.2. Silicate and Sierpiński Graphs. Inorganic networks based on silicates and fractal types, three-dimensional metal-catecholate frameworks, metal-organic frameworks, and reticular chemistry as a whole are emerging as cutting-edge fields of research in catalysis and ultrahigh proton conductivity. The silicate-related $[12,31,32]$ and Imran SabeelE-Hafi [33-35] networks are depicted in Figures 3-5.

The removal of silicon vertices (solid) from the silicaterelated networks resulting with oxide type networks are shown in Figures 6-8.

Recently, $W_{P}$ of oxide OX $(n)$ and silicate SL( $\left.n\right)$ frameworks of dimension $n$ have been computed in [12] using the third neighborhood of vertices as $W_{p}(\mathrm{OX}(n))=63 n^{2}-87 n-$ 3 and $W_{p}(\mathrm{SL}(n))=153 n^{2}-99 n-3$. Now, one can easily obtain these two results by putting $k=1$ and 2 , respectively, in Corollary 1 . We use $\operatorname{DSL}(n), \operatorname{RTSL}(n), \operatorname{DOX}(n)$, and $\operatorname{RTOX}(n)$ to represent the $n$-dimensional dominating silicate, regular triangulene silicate, dominating oxide, and regular triangulene oxide networks, respectively. We now recall the Zagreb indices of silicate- and oxide-related structures in Table 2 .
We noted that the Zagreb indices of the dominating silicate network were computed with errors in [31] and can be readily corrected from Table 3 .

Theorem 3. The Wiener polarity indices of silicate- and oxide-related structures are given by the following:
(1) $W_{P}(D S L(n))=459 n^{2}-657 n+249$
(2) $W_{P}(\operatorname{RTSL}(n))=(1 / 2)\left(51 n^{2}+3 n-36\right)$
(3) $W_{P}(\operatorname{DOX}(n))=189 n^{2}-267 n+99$
(4) $W_{P}(R T O X(n))=(1 / 2)\left(21 n^{2}+3 n-16\right)$

Proof. Since silicate- and oxide-related structures are pentagons-free chemical graphs, we have $N_{p}(G)=0$. Moreover, the end vertices of any edge in $\operatorname{DSL}(n)$ and $\operatorname{RTSL}(n)$ have exactly two common neighbors, whereas $\operatorname{DOX}(n)$ and $\operatorname{RTOX}(n)$ have one common neighbor. We can easily see that the number of hexagons in $\operatorname{DSL}(n)$ and $\operatorname{DOX}(n)$ are equal and the same truth for $\operatorname{RTSL}(n)$ and $\operatorname{RTOX}(n)$ structures i.e., $N_{h}(\operatorname{DSL}(n))=N_{h}(\operatorname{DOX}(n))=9 n^{2}-15 n+7$ and $N_{h}(R T S L(n))=N_{h}(R T O X(n))=n(n-1) / 2$. Based on the above values and by Table 2 , we compute the Wiener polarity indices as follows:

$$
\begin{aligned}
W_{P}(\operatorname{DSL}(n)) & =M_{2}(\operatorname{DSL}(n))-3 M_{1}(\operatorname{DSL}(n))+9|E(\operatorname{DSL}(n))|-3 N_{h}(\operatorname{DSL}(n)) \\
& =2916 n^{2}-3456 n+1242-3\left(1134 n^{2}-1242 n+432\right)+9\left(108 n^{2}-108 n+36\right)-3\left(9 n^{2}-15 n+7\right) \\
& =459 n^{2}-657 n+249 . \\
W_{P}(\operatorname{RTSL}(n)) & =M_{2}(\operatorname{RTSL}(n))-3 M_{1}(\operatorname{RTSL}(n))+9|E(\operatorname{RTSL}(n))|-3 N_{h}(\operatorname{RTSL}(n)) \\
& =162 n^{2}+189 n-72-3\left(63 n^{2}+99 n-18\right)+9\left(6 n^{2}+12 n\right)-3\left(9 n^{2}-15 n+7\right), \\
& =\frac{1}{2}\left(51 n^{2}+3 n-36\right) . \\
W_{P}(\operatorname{DOX}(n))= & M_{2}(\operatorname{DOX}(n))-2 M_{1}(\operatorname{DOX}(n))+4|E(\operatorname{DOX}(n))|-3 N_{h}(\operatorname{DOX}(n)), \\
= & 864 n^{2}-1056 n+384-3\left(432 n^{2}-480 n+168\right)+9\left(54 n^{2}-54 n+18\right)-\frac{3 n(n-1)}{2}, \\
= & 189 n^{2}-267 n+99 . \\
= & \frac{1}{2}\left(21 n^{2}+3 n-16\right) . \\
W_{P}(\operatorname{RTOX}(n))= & 48 n^{2}+48 n-24-3\left(24 n^{2}+36 n-8\right)+9\left(3 n^{2}+6 n\right)-\frac{3 n(n-1)}{2}, \\
&
\end{aligned}
$$

We now give the brief definitions of Sierpiński and its gasket graphs and followed by computations of $W_{P}$. The 1-dimensional Sierpiński graph $S_{1}$ is a complete graph on three vertices, and an $n$-dimensional Sierpiński graph $S_{n}$ is constructed by connecting three copies of $S_{n-1}$ with three bringing edges, as shown in Figure 9. 

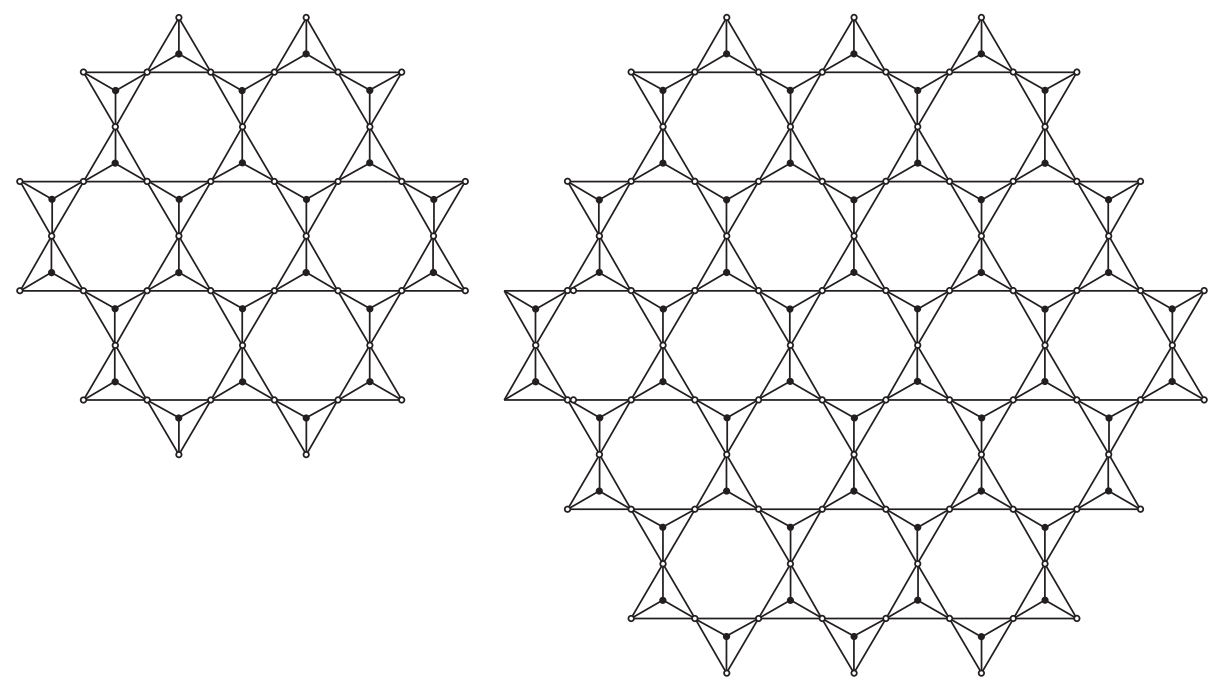

(a)

(b)

Figure 3: (a) Silicate SL(2); (b) silicate SL(3).

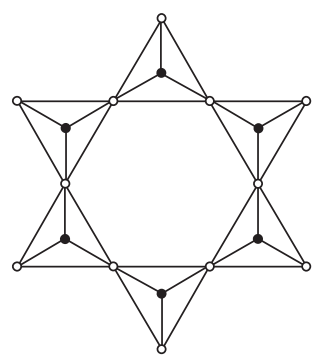

(a)

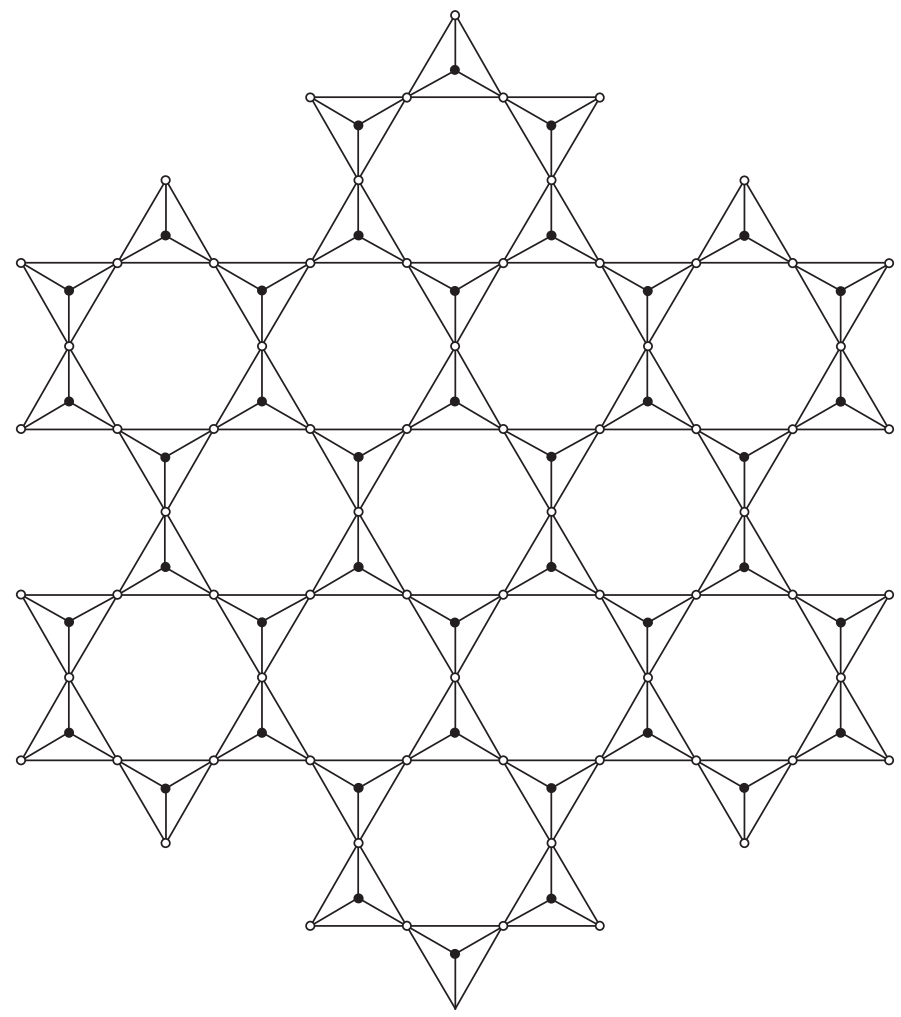

(b)

FIgURE 4: (a) Dominating silicate DSL(1); (b) dominating silicate DSL(2).

The Sierpiński gasket $\mathrm{ST}_{n}$ is obtained from $S_{n}$ by contracting all its bridging edges (Figure 10). The first and second Zagreb indices of $S_{n}$ and $\mathrm{ST}_{n}$ were computed in [37], and $(a, b)$-Zagreb index was dealt in [38]. The number of vertices and edges of $S_{n}$ are $3^{n}$ and $\left(3^{n+1}-3\right) / 2$, respectively. Similarly, $\mathrm{ST}_{n}$ comprises of $\left(3^{n}+3\right) / 2$ vertices and $3^{n}$ edges.
Theorem 4. For $n \geq 3$, the Wiener polarity indices of Sierpinski graph and the Sierpinski gasket are given by the following:

(1) $W_{P}\left(S_{n}\right)=8 \times 3^{n-1}-12$

(2) $W_{P}\left(S T_{n}\right)=26 \times 3^{n-2}-24$ 

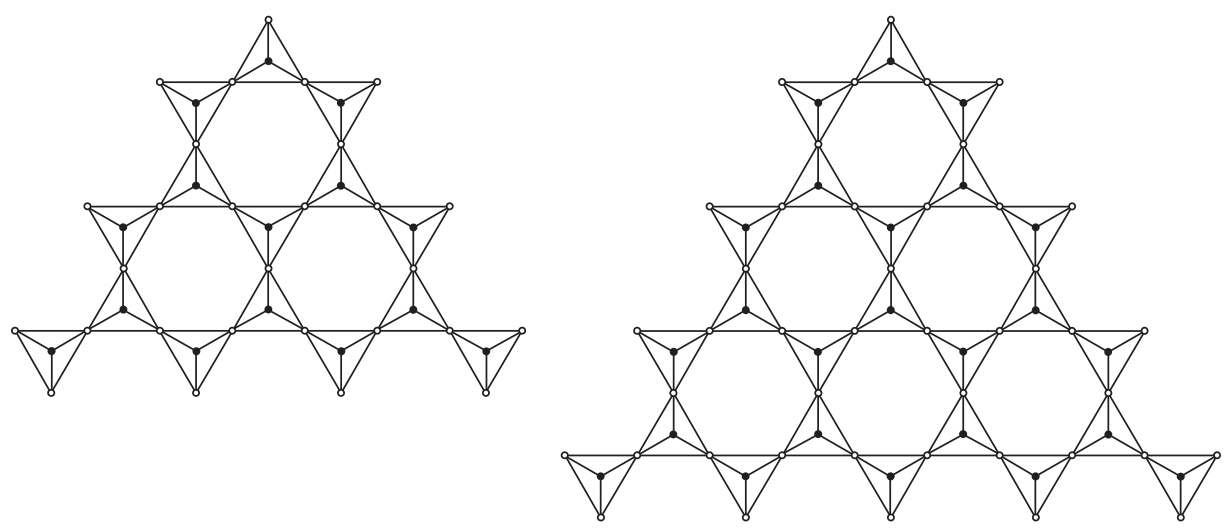

(a)

(b)

Figure 5: (a) Regular triangulene silicate RTSL(3); (b) regular triangulene silicate RTSL(4).
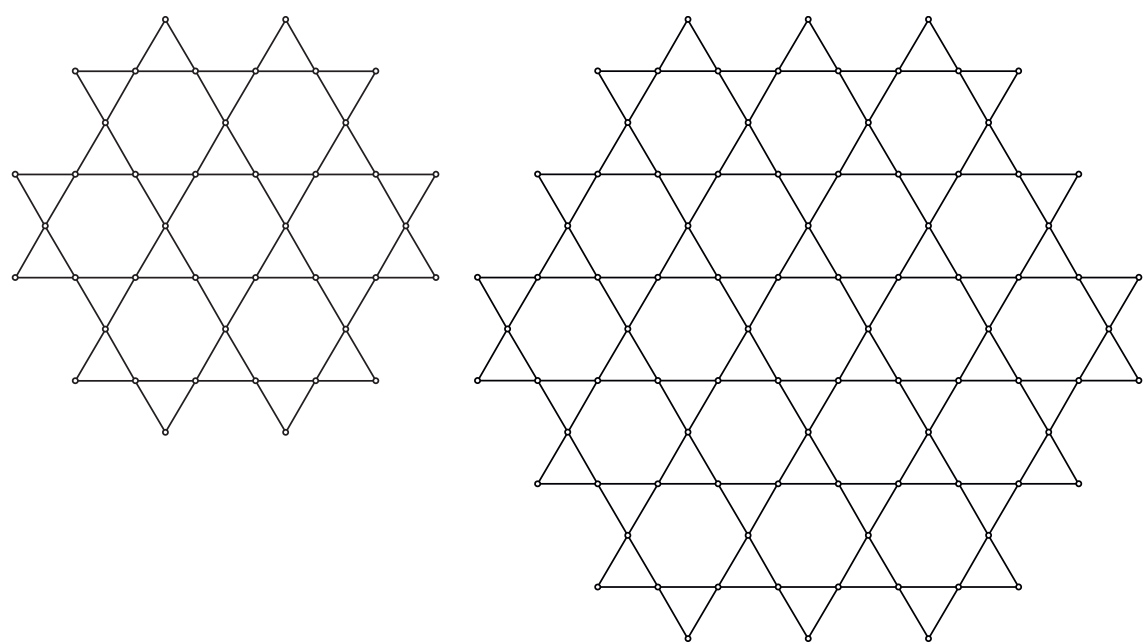

(a)

(b)

Figure 6: (a) Oxide OX(2); (b) oxide OX(3).

Proof. We complete the proof by using Theorem 1 and can be obtained similarly. Let $E_{i j}=\left\{u v \in E\left(S_{n}\right) ; d_{u}=\right.$ Table 4. Now, we derive the first formula, and the second one $\left.i, d_{v}=j\right\}$ :

$$
\begin{aligned}
W_{P}\left(S_{n}\right)= & \sum_{u v \in E\left(S_{n}\right)}\left(d_{u}-k_{u v}-1\right)\left(d_{v}-k_{u v}-1\right)-5 N_{p}\left(S_{n}\right)-3 N_{h}\left(S_{n}\right), \\
= & \sum_{u v \in E_{33} k_{u v}=0}\left(d_{u}-k_{u v}-1\right)\left(d_{v}-k_{u v}-1\right)+\sum_{u v \in E_{33} k_{u v}=1}\left(d_{u}-k_{u v}-1\right)\left(d_{v}-k_{u v}-1\right) \\
& +\sum_{u v \in E_{23} k_{u v}=1}\left(d_{u}-k_{u v}-1\right)\left(d_{v}-k_{u v}-1\right)-5 N_{p}\left(S_{n}\right)-3 N_{h}\left(S_{n}\right), \\
= & 4\left(\frac{3^{n}-3}{2}\right)+3^{n}-6-3\left(3^{n-2}\right), \\
= & 8 \times 3^{n-1}-12 .
\end{aligned}
$$



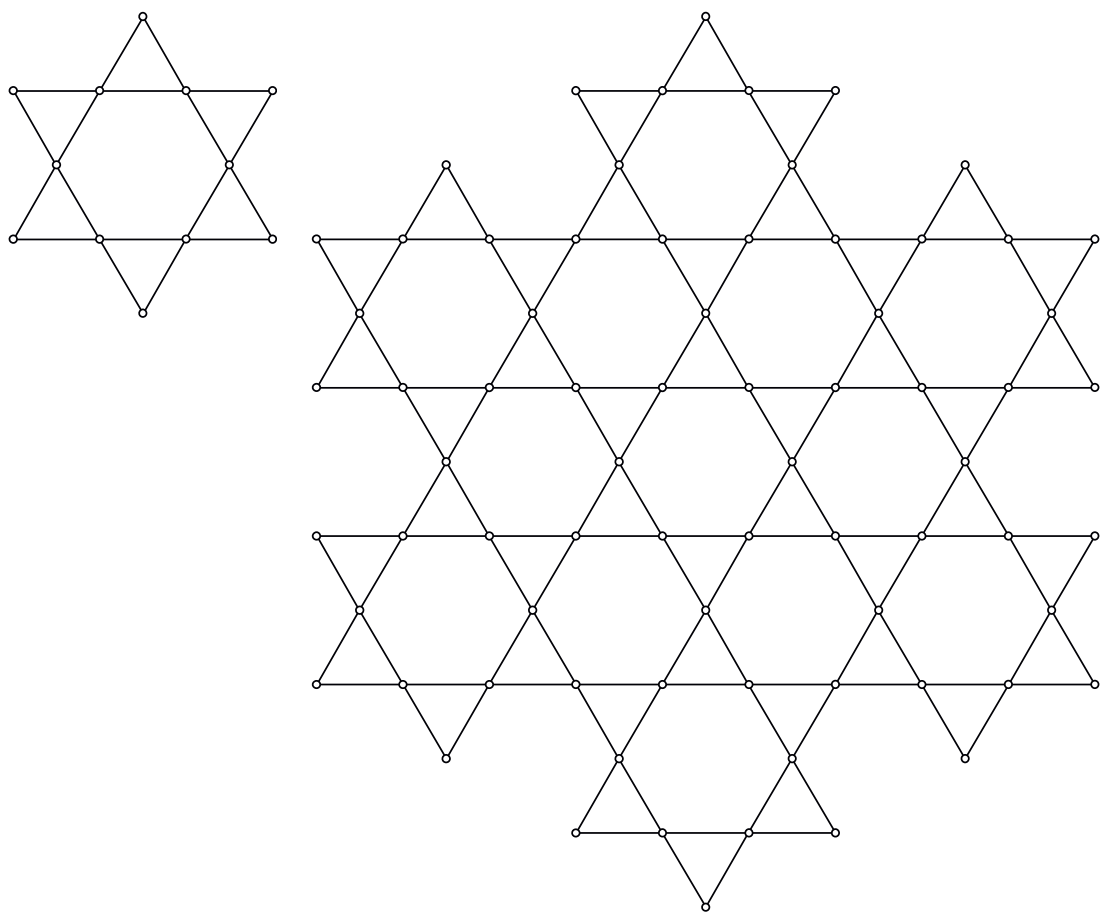

(a)

(b)

Figure 7: (a) Dominating oxide DOX(1); (b) dominating oxide DOX(2).

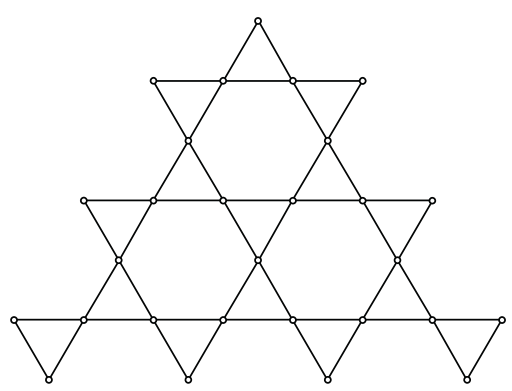

(a)

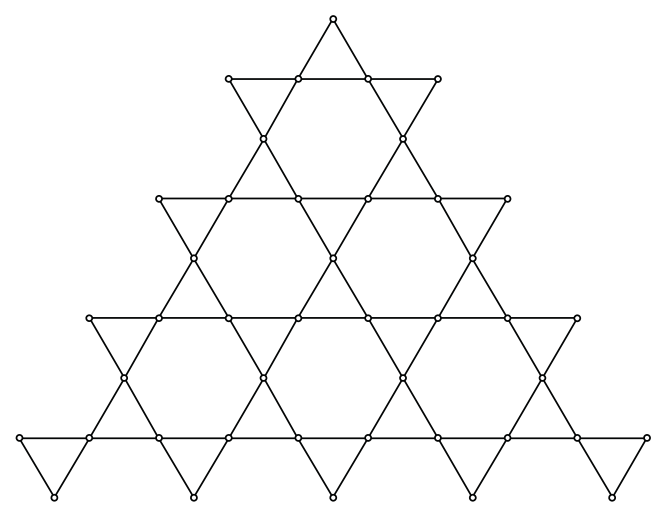

(b)

Figure 8: (a) Regular triangulene oxide RTOX(3); (b) regular triangulene oxide RTOX(4).

TABle 2: Number of edges and Zagreb indices of silicate- and oxide-related structures.

\begin{tabular}{lccc}
\hline$G$ & $E(G)$ & $M_{1}(G)$ & $M_{2}(G)$ \\
\hline $\operatorname{DSL}(n)$ & $108 n^{2}-108 n+36$ & $1134 n^{2}-1242 n+432$ & $2916 n^{2}-3456 n+1242$ \\
$\operatorname{RTSL}(n)[36]$ & $6 n^{2}+12 n$ & $63 n^{2}+99 n-18$ & $162 n^{2}+189 n-72$ \\
$\operatorname{DOX}(n)[31]$ & $54 n^{2}-54 n+18$ & $432 n^{2}-480 n+168$ & $864 n^{2}-1056 n+384$ \\
$\operatorname{RTOX}(n)[31]$ & $3 n^{2}+6 n$ & $24 n^{2}+36 n-8$ & $48 n^{2}+48 n-24$ \\
\hline
\end{tabular}

TABLE 3: Edge partition of $\operatorname{DSL}(n)$.

\begin{tabular}{lccr}
\hline$G$ & Edge type $E_{i}$ & $\left(d_{u}, d_{v}\right) ; u v \in E_{i}$ & $E_{i}$ \\
\hline \multirow{2}{*}{$(n)$} & 1 & $(3,3)$ & $12 n-6$ \\
& 2 & $(3,6)$ & $54 n^{2}-42 n+12$ \\
& 3 & $(6,6)$ & $54 n^{2}-78 n+30$ \\
\hline
\end{tabular}



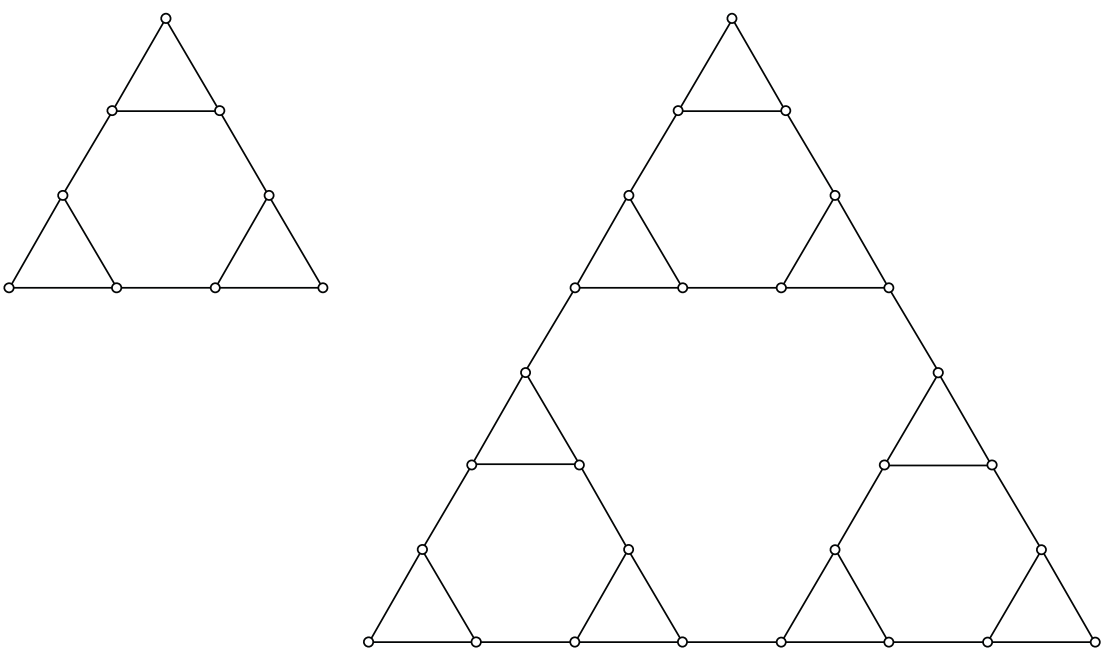

(a)

(b)

FiguRE 9: (a) Sierpiński graph $S_{2}$; (b) Sierpiński graph $S_{3}$.
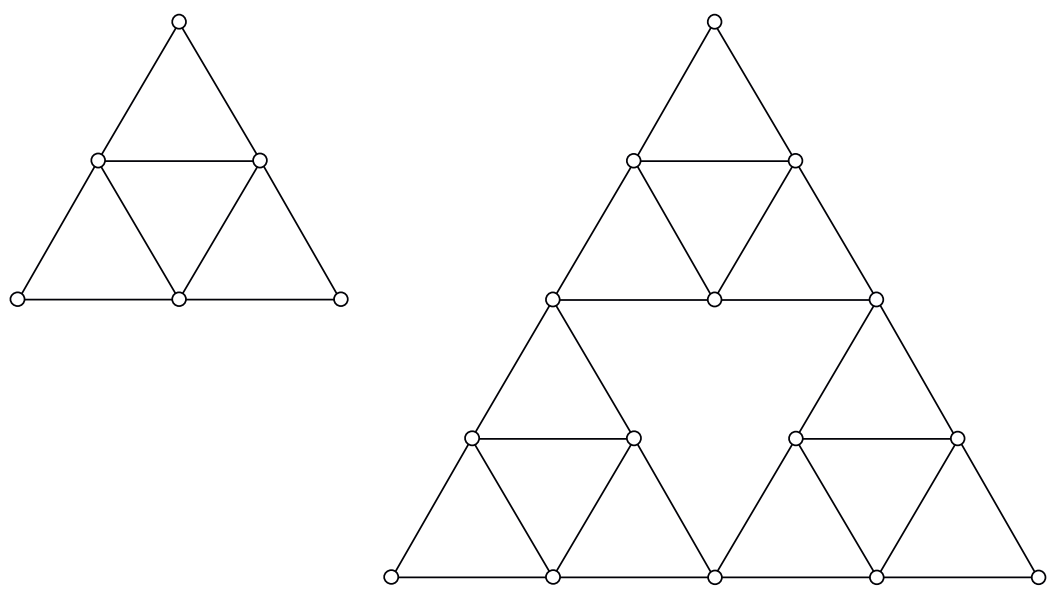

(a)

(b)

FIGURE 10: (a) Sierpiński gasket $\mathrm{ST}_{2}$; (b) Sierpiński gasket $\mathrm{ST}_{3}$.

TABLE 4: The number of vertices, edges, pentagons, hexagons, and edge set partition of $S_{n}$ and $\mathrm{ST}_{n}$.

\begin{tabular}{|c|c|c|c|c|c|}
\hline G & $N_{p}(G)$ & $N_{h}(G)$ & Edge partition $\left(d_{u}, d_{v}\right) ; u v \in E(G)$ & $k_{u v}$ & $\left|E_{i j}\right|$ \\
\hline \multirow{3}{*}{$S_{n}$} & \multirow{3}{*}{0} & \multirow{3}{*}{$3^{n-2}$} & \multirow{2}{*}{$(3,3)$} & 0 & $\left(3^{n}-3\right) / 2$ \\
\hline & & & & 1 & $3^{n}-6$ \\
\hline & & & $(2,3)$ & 1 & 6 \\
\hline \multirow{3}{*}{$\mathrm{ST}_{n}$} & \multirow{3}{*}{0} & \multirow{3}{*}{$3^{n-3}$} & \multirow{3}{*}{$(4,4)$} & 1 & $2\left(3^{n-1}-3\right)$ \\
\hline & & & & 2 & $3^{n-1}$ \\
\hline & & & & 1 & 6 \\
\hline
\end{tabular}

3.3. Octahedral Structures. The idea of octahedral coordination geometry was created by Alfred Werner in 1913 for which he was awarded the Nobel Prize in Chemistry [39]. He clarified the stoichiometries and isomerism in the coordination mix using octahedral coordination geometry. His understanding permitted scientists to legitimize the number of isomers of coordination mixes. Octahedral progress metal buildings containing amines and basic anions are regularly alluded to as Werner-type edifices.

In chemistry, octahedral molecular geometry portrays the shape of compounds with six atoms or gatherings of atoms or ligands symmetrically arranged around a focal 


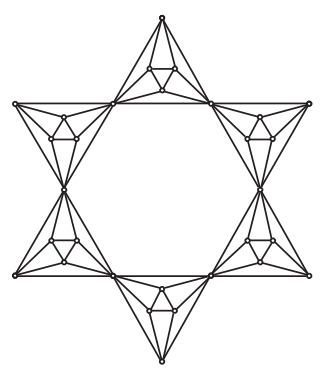

(a)

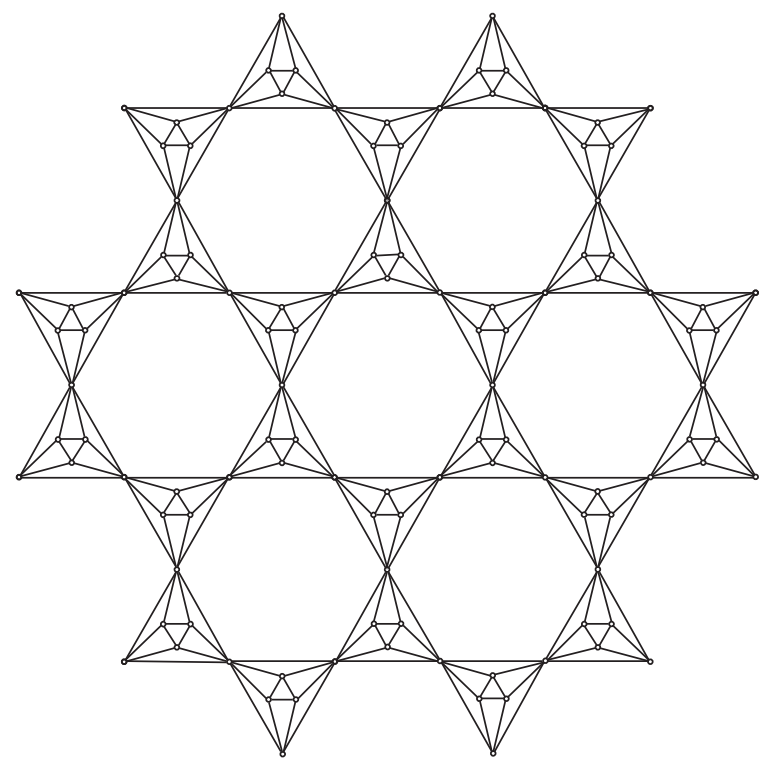

(b)

Figure 11: Octahedral network. (a) OT(1); (b) OT(2).
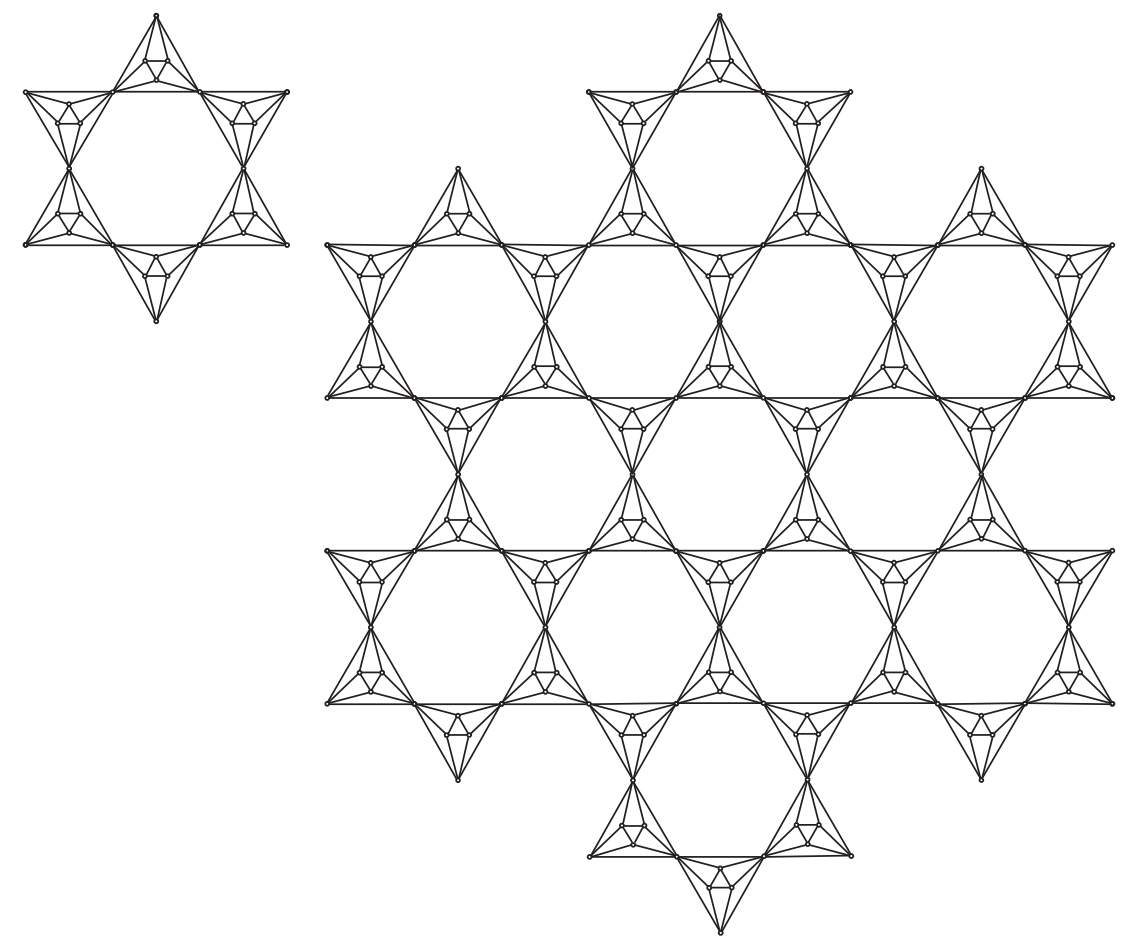

(a)

(b)

Figure 12: Dominated octahedral network. (a) DOT(1); (b) DOT(2).

atom, characterizing the vertices of an octahedron. The octahedron is one of the platonic solids, even though octahedral molecules commonly have an atom in their centre and no bonds between the ligand atoms. A perfect octahedron fits the point group $\mathrm{OH}$. Illustrations of octahedral compounds are sulfur hexafluoride $\mathrm{SF}_{6}$ and molybdenum hexacarbonyl $\mathrm{Mo}(\mathrm{CO})_{6}$. The term octahedral is used somewhat lightly by chemists, concentrating on the geometry of the bonds to the central atom and not considering modifications between the ligands themselves.

The octahedral network was very recently introduced in [40]. Here, we extend the network to its rectangular form 


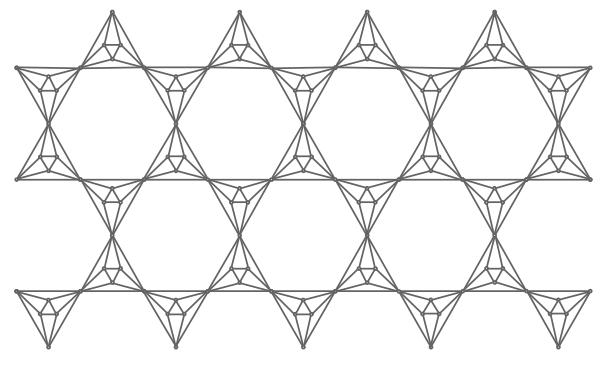

(a)

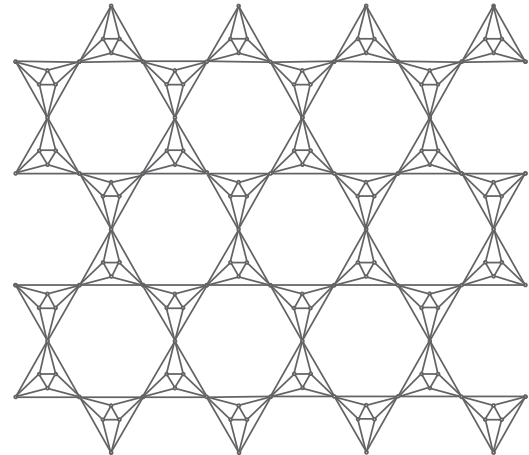

(b)

Figure 13: Rectangular octahedral network. (a) $\operatorname{ROH}(3,9)$; (b) $\operatorname{ROH}(4,8)$.

TABLE 5: Edge partition of DOT $(n)$.

\begin{tabular}{lccc}
\hline$G$ & $\left(d_{u}, d_{v}\right) ; u v \in E(G)$ & $\left|E_{i, j}\right|$ & $N_{h}(G)$ \\
\hline & $(4,4)$ & $54 n^{2}-30 n+6$ & $5 n^{2}-78 n+30$ \\
$\operatorname{DOT}(n)$ & $(8,8)$ & $108 n^{2}-108 n+36$ & $9 n^{2}-15 n+7$ \\
\hline
\end{tabular}

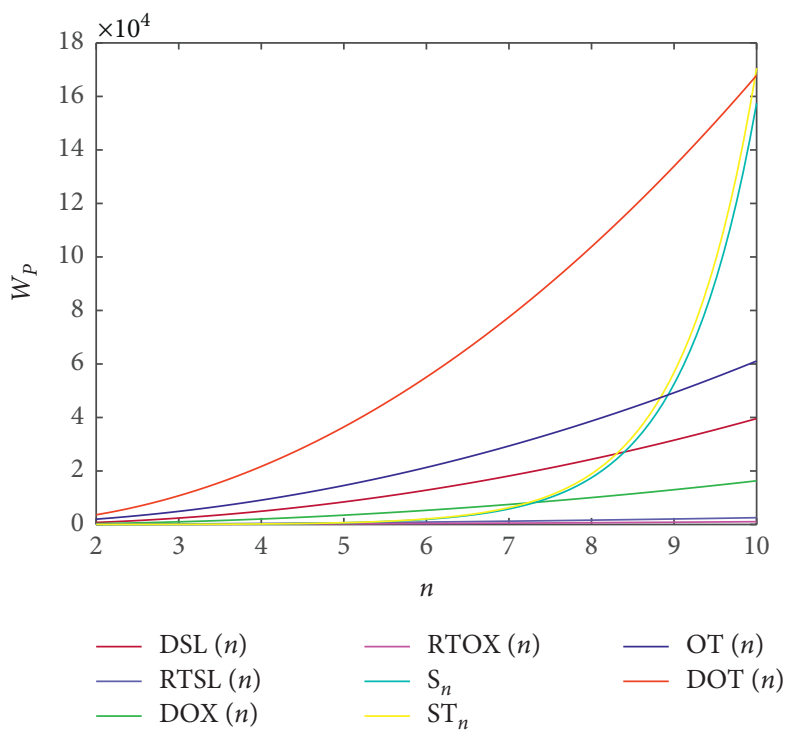

Figure 14: A graphical comparison of Wiener polarity indices of silicate, oxide, Sierpiński, and octahedral-derived structures.

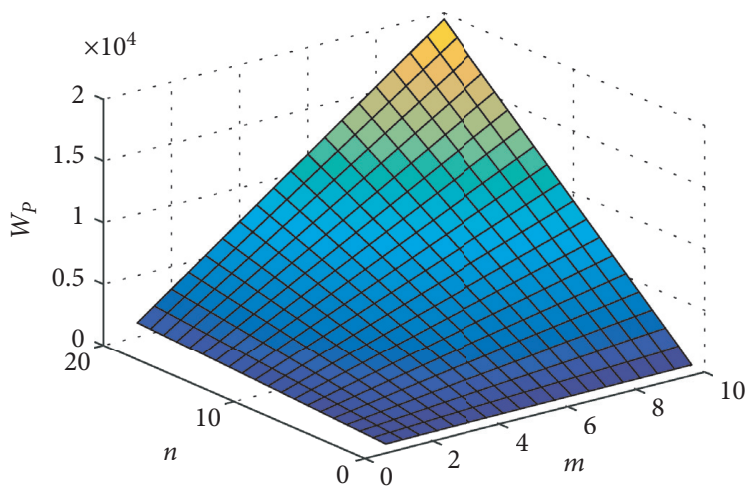

FigURE 15: A graphical representation of the Wiener polarity index of rectangular octahedral networks. 
with the help of the idea adopted in [41]. The octahedral network and its dominated version are depicted, respectively, in Figures 11 and 12, whereas the rectangular form is portrayed in Figure 13.

Theorem 5. The Wiener polarity indices of octahedral $O T(n)$ and dominating octahedral DOT $(n)$ networks are given by the following:

(1) $W_{P}(O T(n))=639 n^{2}-279 n-3$
(2) $W_{P}(\operatorname{DOT}(n))=1917 n^{2}-2475 n+915$

Proof. The Zagreb indices of OT $(n)$ have been obtained [40] as $M_{1}(\mathrm{OT}(n))=96 n(9 n-1)$ and $M_{2}(\mathrm{OT}(n))=288 n$ $(9 n-2)$. Using Table 5, we can easily derive the Zagreb indices of $\operatorname{DOT}(n)$ as $M_{1}(\operatorname{DOT}(n))=2592 n^{2}-2784 n+$ 960 and $M_{2}(\operatorname{DOT}(n))=7776 n^{2}-8928 n+3168$. Moreover, the end vertices of any edge in $\mathrm{OT}(n)$ and $\operatorname{DOT}(n)$ have exactly two common neighbors, and we have

$$
\begin{aligned}
W_{P}(\mathrm{OT}(n)) & =M_{2}(\mathrm{OT}(n))-3 M_{1}(\mathrm{OT}(n))+9|E(\mathrm{OT}(n))|-3 N_{h}(\mathrm{OT}(n)), \\
& =288 n(9 n-2)-3(96 n(9 n-1))+9\left(72 n^{2}\right)-3\left(3 n^{2}-3 n+1\right), \\
& =639 n^{2}-279 n-3 . \\
W_{P}(\operatorname{DOT}(n)) & =M_{2}(\operatorname{DOT}(n))-3 M_{1}(\operatorname{DOT}(n))+9|E(\operatorname{DOT}(n))|-3 N_{h}(\operatorname{DOT}(n)), \\
& =7776 n^{2}-8928 n+3168-3\left(2592 n^{2}-2784 n+960\right)+9\left(216 n^{2}-216 n+72\right)-3\left(9 n^{2}-15 n+7\right), \\
& =1917 n^{2}-2475 n+915 .
\end{aligned}
$$

Theorem 6. The Zagreb and Wiener polarity indices of rectangular octahedral $\operatorname{ROT}(m, n)$ networks are given by the following:

(1) $M_{1}(R O T(m, n))=\{144 m n-32 m-16 n+16$, : $m$ even and $n$ odd, $144 m n-32 m-16 n$, : otherwise.

(2) $M_{2}(R O T(m, n))=\{432 m n-192 m-96 n+96$, : meven and $n$ odd, $432 m n-192 m-96 n+$ 32 , : otherwise.
(3) $W_{P}(\operatorname{ROT}(m, n))=\{(1 / 2)(213 m n-186 m-93 n+$ $90),:$ m even and $n$ odd,$(1 / 2)(213 m n-186 m-$ $93 n+58)$, : otherwise.

Proof. For $\operatorname{ROT}(m, n), m$ even and $n$ odd, we have $E(\operatorname{ROT}(m, n))=E_{4,4} \cup E_{4,8} \cup E_{8,8}$ where $\left|E_{4,4}\right|=3 m n+4 m+$ $2 n-2,\left|E_{4,8}\right|=6 m n$, and $\left|E_{8,8}\right|=3 m n-4 m-2 n+2$. In this case, the number of hexagon in $\operatorname{ROT}(m, n)$ is $(1 / 2)(m n-2 m-n+3)$, and the end vertices of any edge has exactly two common neighbors:

$$
\begin{aligned}
M_{1}(\operatorname{ROT}(m, n)) & =\sum_{u v \in E_{4,4}}\left(d_{u}+d_{v}\right)+\sum_{u v \in E_{4,8}}\left(d_{u}+d_{v}\right)+\sum_{u v \in E_{8,8}}\left(d_{u}+d_{v}\right), \\
& =8(3 m n+4 m+2 n-2)+12(6 m n)+16(3 m n-4 m-2 n+2), \\
& =144 m n-32 m-16 n+16 . \\
M_{2}(\operatorname{ROT}(m, n)) & =\sum_{u v \in E_{4,4}}\left(d_{u} \times d_{v}\right)+\sum_{u v \in E_{4,8}}\left(d_{u} \times d_{v}\right)+\sum_{u v \in E_{8,8}}\left(d_{u} \times d_{v}\right), \\
& =16(3 m n+4 m+2 n-2)+32(6 m n)+64(3 m n-4 m-2 n+2), \\
& =432 m n-192 m-96 n+96 .
\end{aligned}
$$

$W_{P}(\operatorname{ROT}(m, n))=M_{2}(\operatorname{ROT}(m, n))-(k+1) M_{1}(\operatorname{ROT}(m, n))+(k+1)^{2}|E(\operatorname{ROT}(m, n))|-3 N_{h}(\operatorname{ROT}(m, n))$

$$
\begin{aligned}
& =432 m n-192 m-96 n+96-3(144 m n-32 m-16 n+16)+9(12 m n)-\frac{3}{2}(m n-2 m-n+3), \\
& =\frac{1}{2}(213 m n-186 m-93 n+90) .
\end{aligned}
$$


For all other values of $m$ and $n$ in $\operatorname{ROT}(m, n)$, we have $\left|E_{4,4}\right|=3 m n+4 m+2 n+2,\left|E_{4,8}\right|=6 m n-4,\left|E_{8,8}\right|=3 m n-$
$4 m-2 n+2$, and $N_{h}(\operatorname{ROT}(m, n))=(1 / 2)(m n-2 m-n+$ 2). Then,

$$
\begin{aligned}
M_{1}(\operatorname{ROT}(m, n)) & =\sum_{u v \in E_{4,4}}\left(d_{u}+d_{v}\right)+\sum_{u v \in E_{4,8}}\left(d_{u}+d_{v}\right)+\sum_{u v \in E_{8,8}}\left(d_{u}+d_{v}\right), \\
& =8(3 m n+4 m+2 n+2)+12(6 m n-4)+16(3 m n-4 m-2 n+2), \\
& =144 m n-32 m-16 n . \\
M_{2}(\operatorname{ROT}(m, n))= & \sum_{u v \in E_{4,4}}\left(d_{u} \times d_{v}\right)+\sum_{u v \in E_{4,8}}\left(d_{u} \times d_{v}\right)+\sum_{u v \in E_{8,8}}\left(d_{u} \times d_{v}\right), \\
= & 16(3 m n+4 m+2 n+2)+32(6 m n-4)+64(3 m n-4 m-2 n+2), \\
= & 432 m n-192 m-96 n+32 . \\
W_{P}(\operatorname{ROT}(m, n))= & M_{2}(\operatorname{ROT}(m, n))-(k+1) M_{1}(\operatorname{ROT}(m, n))+(k+1)^{2}|E(\operatorname{ROT}(m, n))|-3 N_{h}(\operatorname{ROT}(m, n)), \\
= & 432 m n-192 m-96 n+32-3(144 m n-32 m-16 n)+9(12 m n)-\frac{3}{2}(m n-2 m-n+2), \\
= & \frac{1}{2}(213 m n-186 m-93 n+58) .
\end{aligned}
$$

We have shown the graphical plots of our computed results in Figures 14 and 15.

\section{Conclusion}

In this paper, we have derived the technique to find the Wiener polarity indices of graphs without squares, and consequently, we have computed the Wiener polarity indices of chemical structures of lenvatinib and cabozantinib, which are used in the treatment of thyroid cancer and HCC. As measured topological indices are proficient at forecasting different properties and behaviors such as boiling point, entropy, enthalpy, and critical pressure, our results can be useful in designing new drugs and vaccines for cancer. In addition to this, we have computed the Wiener polarity indices of some special classes of graphs, namely, silicate, Sierpiński, and octahedral structures with the help of our extended result.

\section{Data Availability}

The figures, tables, and other data used to support the findings of this study are included within the article.

\section{Conflicts of Interest}

The authors declare that they have no conflicts of interest regarding the publication of this paper.

\section{References}

[1] S.-F. Zhou and W.-Z. Zhong, "Drug design and discovery: principles and applications," Molecules, vol. 22, no. 2, p. 279, 2017.

[2] K. Balasubramanian, "Mathematical and computational techniques for drug discovery: promises and developments," Current Topics in Medicinal Chemistry, vol. 18, no. 32, pp. 2774-2799, 2018.

[3] W. Gao, Y. Wang, B. Basavanagoud, and M. K. Jamil, "Characteristics studies of molecular structures in drugs," Saudi Pharmaceutical Journal, vol. 25, no. 4, pp. 580-586, 2017.

[4] J. Devillers and A. T. Balaban, Topological Indices and Related Descriptors in QSAR and QSPR, Gordon \& Breach, Amsterdam, Netherlands, 1999.

[5] H. Wiener, "Structural determination of paraffin boiling points," Journal of the American Chemical Society, vol. 69, no. 1, pp. 17-20, 1947.

[6] J. B. Liu, J. Zhao, and J. Min, "On the Hosoya index of graphs formed by a fractal graph," Fractals, vol. 27, no. 8, pp. 19-35, 2019.

[7] J.-B. Liu, J. Zhao, H. He, and Z. Shao, "Valency-based topological descriptors and structural property of the generalized Sierpiński networks," Journal of Statistical Physics, vol. 177, no. 6, pp. 1131-1147, 2019.

[8] J. B. Liu, J. Zhao, and Z. Cai, "On the generalized adjacency, Laplacian and signless Laplacian spectra of the weighted edge corona networks," Physica A, vol. 540, pp. 12-30, 2020.

[9] W. Fang, M. Ma, F. Chen, and H. Dong, "Third smallest Wiener polarity index of unicyclic graphs," Frontiers in Physics, vol. 8, 2020. 
[10] M. Alaeiyan, F. Afzal, M. R. Farahani, and M. A. Rostami, "An exact formulas for the Wiener polarity index of nanostar dendrimers," Journal of Information and Optimization Sciences, vol. 41, no. 4, p. 933, 2020.

[11] L. Chen, T. Li, J. Liu, Y. Shi, and H. Wang, "On the Wiener polarity index of lattice networks," PLoS One, vol. 11, no. 12, Article ID e0167075, 2016.

[12] M. Arockiaraj, S. R. J. Kavitha, K. Balasubramanian, and I. Gutman, "Hyper-Wiener and Wiener polarity indices of silicate and oxide frameworks," Journal of Mathematical Chemistry, vol. 56, no. 5, pp. 1493-1510, 2018.

[13] A. R. Ashrafi and A. Ghalavand, "Ordering chemical trees by Wiener polarity index," Applied Mathematics and Computation, vol. 313, pp. 301-312, 2017.

[14] A. Behmaram, H. Yousefi-Azari, and A. R. Ashrafi, "Wiener polarity index of fullerenes and hexagonal systems," Applied Mathematics Letters, vol. 25, no. 10, pp. 1510-1513, 2012.

[15] H. Deng, H. Xiao, and F. Tang, "On the extremal Wiener polarity index of trees with a given diameter," MATCH Communications in Mathematical and in Computer Chemistry, vol. 63, no. 1, pp. 257-264, 2010.

[16] H. Deng and H. Xiao, "The Wiener polarity index of molecular graphs of alkanes with a given number of methyl groups," Journal of the Serbian Chemical Society, vol. 75, no. 10, pp. 1405-1412, 2010.

[17] H. Deng, "On the extremal Wiener polarity index of chemical trees," MATCH Communications in Mathematical and in Computer Chemistry, vol. 66, no. 1, pp. 305-314, 2011.

[18] W. Du, X. Li, and Y. Shi, "Algorithms and extremal problem on Wiener polarity index," MATCH Communications in Mathematical and in Computer Chemistry, vol. 62, no. 1, pp. 235-244, 2009.

[19] H. Hosoya, "Mathematical and chemical analysis of Wiener's polarity number," in Topology in Chemistry: Discrete Mathematics of Molecules, D. H. Rouvray and R. B. King, Eds., Horwood, Chichester, UK, 2002.

[20] H. Hou, B. Liu, and Y. Huang, "The maximum Wiener polarity index of unicyclic graphs," Applied Mathematics and Computation, vol. 218, no. 20, pp. 10149-10157, 2012.

[21] H. Hua and K. C. Das, "On the Wiener polarity index of graphs," Applied Mathematics and Computation, vol. 280, pp. 162-167, 2016.

[22] A. Ilić and M. Ilić, "Generalizations of Wiener polarity index and terminal Wiener index," Graphs and Combinatorics, vol. 29, no. 5, pp. 1403-1416, 2013.

[23] G. Liu and G. Liu, "Wiener polarity index of dendrimers," Applied Mathematics and Computation, vol. 322, pp. 151-153, 2018.

[24] B. Liu, H. Hou, and Y. Huang, "On the Wiener polarity index of trees with maximum degree or given number of leaves," Computers \& Mathematics with Applications, vol. 60, no. 7, pp. 2053-2057, 2010.

[25] I. Lukovits and W. Linert, "Polarity-numbers of cycle-containing structures," Journal of Chemical Information and Computer Sciences, vol. 38, no. 4, pp. 715-719, 1998.

[26] J. Ma, Y. Shi, and J. Yue, "The Wiener polarity index of graph products,” Ars Combinatoria, vol. 116, pp. 235-244, 2014.

[27] J. Ma, Y. Shi, Z. Wang, and J. Yue, "On Wiener polarity index of bicyclic networks," Scientific Reports, vol. 6, Article ID 19066, 2016.

[28] Y. Zhang and Y. Hu, "The Nordhaus-Gaddum-type inequality for the Wiener polarity index," Applied Mathematics and Computation, vol. 273, pp. 880-884, 2016.
[29] M. Arockiaraj, J. B. Liu, S. Prabhu, and M. Arulperumjothi, "On the zagreb and wiener polarity indices of $C_{3}$-free chemical nanostructures," Utilitas Mathematica, 2018.

[30] D. Li, S. Sedano, R. Allen, J. Gong, M. Cho, and S. Sharma, "Current treatment landscape for advanced hepatocellular carcinoma: patient outcomes and the impact on quality of life," Cancers, vol. 11, no. 6, p. 841, 2019.

[31] A. Q. Baig, M. Imran, and H. Ali, "On topological indices of poly oxide, poly silicate, DOX, and DSL networks," Canadian Journal of Chemistry, vol. 93, no. 7, pp. 730-739, 2015.

[32] M. Cancan, D. Afzal, S. Hussain, A. Maqbool, and F. Afzal, "Some new topological indices of silicate network via M-polynomial," Journal of Discrete Mathematical Sciences and Cryptography, vol. 23, no. 6, pp. 1157-1171, 2020.

[33] M. Imran Sabeel-E-Hafi, W. Gao, and M. Reza Farahani, "On topological properties of sierpinski networks," Chaos, Solitons \& Fractals, vol. 98, pp. 199-204, 2017.

[34] J. A. Rodríguez-Velázquez and J. Tomás-Andreu, "On the Randić index of polymer networks modelled by generalized Sierpiński," MATCH Communications in Mathematical and in Computer Chemistry, vol. 74, no. 1, pp. 145-160, 2015.

[35] S. Ediz, M. Alaeiyan, M. Alaeiyan, M. Farahani, and M. Cancan, "On Van, $r$ and s topological properties of the Sierpinski triangle networks," Eurasian Chemical Communications, vol. 2, no. 7, p. 819, 2020.

[36] M. Imran, A. Q. Baig, H. Ali, and S. U. Rehman, "On topological properties of poly honeycomb networks," Periodica Mathematica Hungarica, vol. 73, no. 1, pp. 100-119, 2016.

[37] H. M. A. Siddiqui, "Computation of Zagreb indices and Zagreb polynomials of Sierpiński graphs," Hacettepe Journal of Mathematics and Statistics, vol. 49, no. 2, pp. 754-765, 2020.

[38] P. Sarkar, N. De, I. N. Cangul, and A. Pal, "The $(a, b)$-Zagreb index of some derived networks," Journal of Taibah University for Science, vol. 13, no. 1, pp. 79-86, 2020.

[39] E. C. Constable and C. E. Housecroft, "Coordination chemistry: the scientific legacy of Alfred Werner," Chemical Society Reviews, vol. 42, no. 4, pp. 1429-1439, 2013.

[40] M. Arockiaraj, S. R. J. Kavitha, K. Balasubramanian, and J. B. Liu, "On certain topological indices of octahedral and icosahedral networks," IET Control Theory \& Applications, vol. 12, no. 2, pp. 215-220, 2018.

[41] J.-B. Liu, M. K. Shafiq, H. Ali, A. Naseem, N. Maryam, and S. S. Asghar, "Topological indices of $m$ th chain silicate graphs,” Mathematics, vol. 7, no. 1, p. 42, 2019. 